\title{
Environmental RNA interference in animals
}

\author{
MENG LingFeng ${ }^{1}$, CHEN Liang $^{2}$, LI ZhaoYong ${ }^{2}$, WU ZhengXing ${ }^{1 *} \&$ SHAN Ge $^{2 *}$ \\ ${ }^{1}$ School of Life Science and Technology, Huazhong University of Science and Technology, Wuhan 430074, China; \\ ${ }^{2}$ School of Life Sciences \& Key Laboratory of Brain Function and Disease of Chinese Academy of Sciences, University of Science and Technology \\ of China, Hefei 230027, China
}

Received May 12, 2013; accepted June 7, 2013; published online August 21, 2013

\begin{abstract}
Animals interact with their environments all the time, and must react to all kinds of factors present in the environments. Environmental RNA interference refers to the phenomenon that exogenous RNA molecules can enter cells of certain animal species and interfere with gene expression of these animals. These exogenous RNAs may be molecules in animal food, or may be present in the living environment from other sources. Molecular pathways for the cell entry of environmental RNAs and further for their functionality in gene interference have started to be revealed in the animal model Caenorhabditis elegans. Here we highlight some known examples of environmental RNA interference (RNAi) in animals and introduce the molecular mechanisms underneath.
\end{abstract}

environmental RNAi, noncoding RNA, Caenorhabditis elegans, systemic RNAi

Citation: $\quad$ Meng L F, Chen L, Li Z Y, et al. Environmental RNA interference in animals. Chin Sci Bull, 2013, 58: 4418-4425, doi: 10.1007/s11434-013-5982-0

Publication of the landmark article by Andrew Fire, Craig Mello, and colleagues in 1998 has linked double-stranded RNA (dsRNA) with the RNA interference (RNAi) phenomenon [1]. DsRNAs targeting specific gene sequences were injected into Caenorhabditis elegans for this work [1]. Andrew Fire and Craig Mello labs later developed alternative ways for triggering RNAi in C. elegans with exogenous dsRNAs [2,3]. What they did was soaking worms in dsRNA solution or feeding them with recombinant E. coli engineered to express dsRNA (feeding RNAi) [2,3]. These findings indicated that exogenous RNA, specifically dsRNA, could enter cells of $C$. elegans and initiate downstream RNAi effects. The fact that dsRNAs both injected into and fed to worms could trigger a systemic RNAi effect in most cells also indicated the existence of a spreading mechanism for cell to cell transportation/uptake of RNAi signals, presumably also dsRNAs. Actually this kind of environmental and cell nonautonomous RNAi effects have now been found in many organisms including animals, plants, and protozoans [4-6]. In the past decade, molecular pathways for the cell entry of environmental RNAs and the downstream

*Corresponding authors (email: ibbwuzx@mail.hust.edu.cn; shange@ustc.edu.cn)
RNAi effects have been substantially revealed in the animal model $C$. elegans. Efforts have also been made to develop biotechnologies based on the environmental RNAi phenomenon.

\section{Uptake of environmental RNA and intercellular RNA movement in C. elegans}

For environmental RNAi, exogenous RNAs have to be taken up into animal cells. Forward genetic screens with $C$. elegans have identified a number of genes that function in environmental RNAi, and some of them turned out to be involved in the uptaking of dsRNAs from the environments into intestinal cells [7-11] (Figure 1). Among them sid-1 and sid-2 are the first genes cloned functioning in environmental RNAi [4]. sid-1 or sid-2 single mutants lost the ability to trigger RNAi effect in intestinal cells upon feeding RNAi [4]. Actually sid-1 mutant did not exhibit any systemic RNAi response to dsRNA injection, soaking, or feeding, while sid-2 mutant exhibited defect in RNAi by soaking and feeding but remained sensitive to RNAi by injection. sid-1 encodes a multipass transmembrane protein highly 


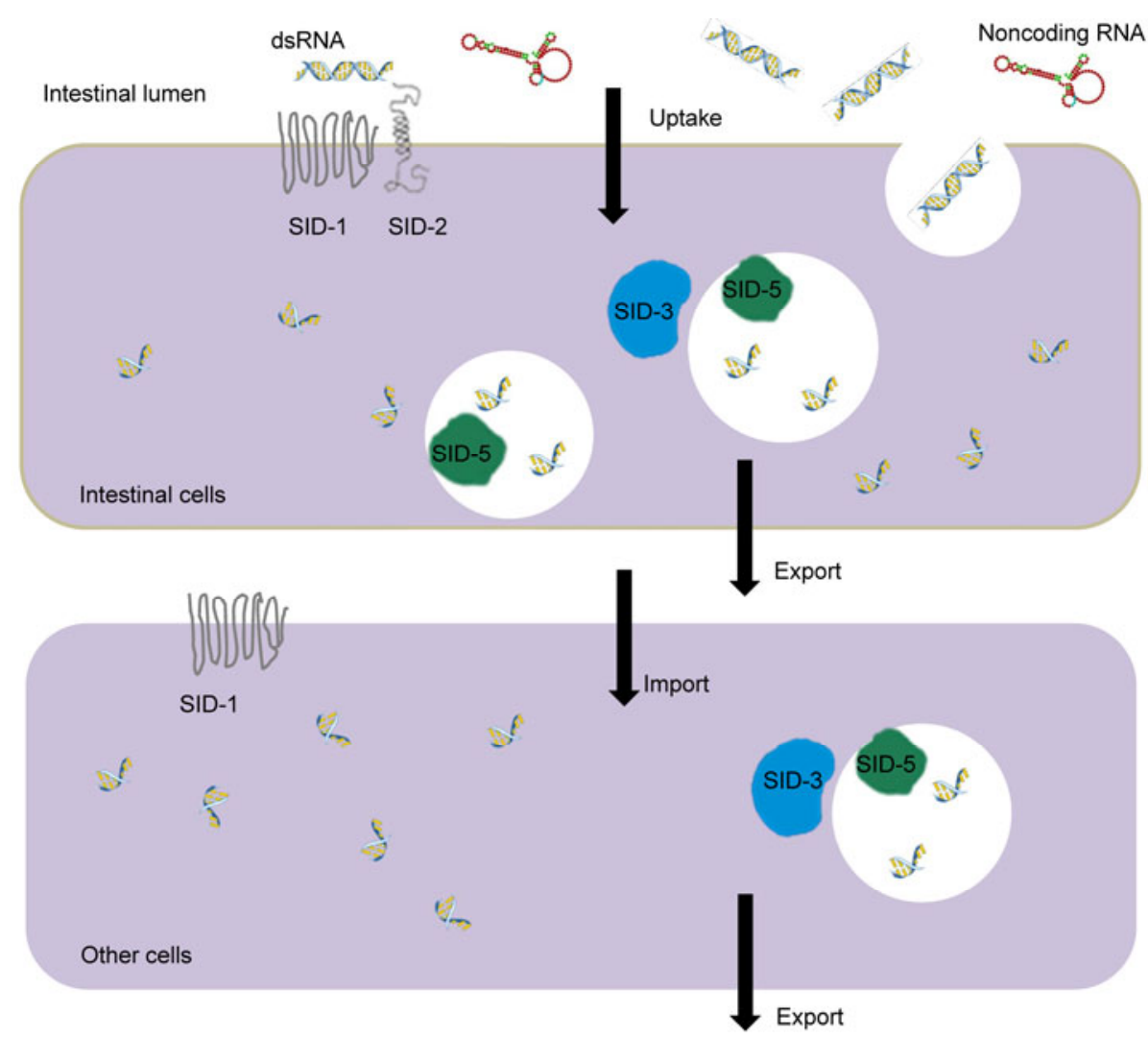

Figure 1 (Color online) Proposed pathway for uptaking, exporting, and importing exogenous environmental RNAs in C. elegans.

conserved from nematode to mammals [7,12]. sid-2 encodes a single-pass transmembrane protein with homologs identified only in two sister species of $C$. elegans, C. briggsae and $C$. remanei [8]. sid- 1 is expressed in most non-neuronal cells including intestinal cells. sid-2 is expressed in intestinal cells, and SID-2::GFP fusion localizes to intestinal cell apical membranes. Heterologous expression of either SID-1 or SID-2 in Drosophila melanogaster S2 cells led to cells gaining the ability of importing dsRNA from culture medium $[12,13]$. According to the structures and functions of SID-1 and SID-2, SID-1 may act as dsRNA channel or a component of channel for uptaking dsRNAs, and SID-2 may act as a dsRNA receptor or a component of receptor to aid the intestinal uptaking by SID-1 of environmental dsRNAs in intestinal lumen. Indeed, it is true that sid-2 is required only for the uptaking of exogenous dsRNAs but is not necessary for the spreading of RNAi signal from intestinal cells to other cells.

Conversely, sid- 1 is required for both the initial import of dsRNA into worms and the spread of systemic RNAi signal, but not necessary for cells to export systemic RNAi signal [14]. SID-2-dependent dsRNA uptaking requires an acidic extracellular environment in intestinal lumen, and is selective for dsRNAs 50 base pairs or longer. SID-2-dependent transport could be suppressed inhibitory drugs of vesicle transportation. Therefore, both acidic environment and endocytosis seem vital in the uptaking of environmental
dsRNA [13].

Reverse genetic screen with $C$. elegans and Drosophila S2 cells had identified multiple genes such as arl-1, vps-34, and vps-41 in the endocytic pathway to be related to environmental RNAi [11]. Recently, sid-3 and sid-5, two environmental RNAi genes related to endocytosis have been cloned by forward genetics $[15,16]$. In sid-3 null mutants RNAi effects triggered by feeding were defective although not abolished [15]. sid-3 encodes a conserved Ack (the activated Cdc-42-associated kinase) protein with a tyrosine kinase domain, a SH3 domain, and a Cdc42/Rac interactive binding domain. It seems that SID-3, a widely expressed cytoplasmic protein, is involved in the importing of silencing signals into cells but not for their exporting out of cells [15]. ACK in mammalian cells is well associated with endocytosis [17], although a direct link between sid-3 with endocytosis in C. elegans is not established yet. sid-5 encodes a worm specific small protein of 67 amino acids with a single predicted transmembrane domain [16]. It is clear that SID-5 protein is colocalized with several late endosomal proteins such as RAB-7 and LMP-1. sid-5 is expressed in essentially all somatic cells, but an intestinal specific expressing of sid-5 could rescue feeding RNAi defect in sid-5 mutants. This result indicated that sid-5 might mainly function to transport silencing signals across intestine by participating in dsRNA export out from intestinal cells and/or dsRNA uptake from the intestinal lumen [16]. 


\section{C. elegans RNAi pathway triggered by exogenous RNA}

Once inside $C$. elegans cells, exogenous dsRNAs follow a similar pathway to endogenous RNAi. Basically these dsRNAs are cut by DCR-1 with the assistance of RDE-4, RDE-1, DRH-1, and DRH-2 [18-22] (Figure 2). RDE-1 then directs the so-called primary siRNA to its targeting mRNA, resulting in the degradation of target mRNA and triggering the amplification of secondary siRNA by RNA dependent RNA polymerases (RdRP) such as RRF-1 and EGO-1 with the involvement of DRH-3 [23-27]. CSR-1 and some other secondary Argonaute proteins (SAGO) may be responsible for the gene silencing effect of secondary siRNA, and a number of factors such as MUT-7/-14/-15/-16, RDE-2/-3, SAGO-1/-2, and PPW-1 may play crucial roles in the effect of secondary siRNA [28-32] (Figure 2).

Nine out of 61 C. elegans ATP-binding cassette (ABC) transporters are also involved in the environmental RNAi effect [33]. Mutants of ABC transporter genes abt-1, pgp-4, pgp-11, haf-2, haf-6, mrp-1, pmp-1, C16C10.12, and C05D10.3 showed strong defective in feeding RNAi [33]. Among these nine genes, haf- 6 was characterized further. haf-6(ne335) displayed dosage sensitive in injection and soaking induced RNAi. Injection or soaking with high concentrations of dsRNA could induce the RNAi effect, while lower concentrations were less effective. haf- 6 mutant also showed defect in intestinal and germline cells but not in other tissues in feeding RNAi. Research had shown that haf-6 was expressed specifically in intestinal cells and germline cells, and HAF-6 protein might localize to endo- plasmic reticulum [33]. All these $9 \mathrm{ABC}$ transporters were later found to be interact genetically for their effects in RNAi with $r d e-2$ and mut-7, two genes known to be involved in the effects of secondary siRNA [34]. Just like haf- 6 mutant, mutants of two genes $r d e-10$ and $r d e-11$ were recently identified to be only sensitive to high dosage of dsRNA [35]. RDE-10, RDE-11, RSD-2, RSD-6, and HAF-6 may function together to promote the accumulation of secondary siRNAs originated from both exogenous and endogenous dsRNAs, although detailed molecular mechanism about exactly how these factors perform their job needs further investigation [34,35] (Figure 2). Secondary siRNAs from both exogenous and endogenous RNAs could also enter cell nucleus to inhibit gene transcription with the function of NRDE-2/-3 complex [36,37] (Figure 2). Exogenous environmental RNAi may also interfere with the endogenous RNAi in C. elegans by competing shared factors such as secondary Argonautes in the RNAi pathway [38].

\section{Natural feeding RNAi in C. elegans}

Essentially all experiments used to elucidate the molecular mechanisms underlying environmental RNAi were with artificially synthesized or recombinantly expressed dsRNAs under the laboratory condition. Investigators have recently found examples of natural feeding RNAi triggered by noncoding RNAs encoded by bacterial food of $C$. elegans. Ge Shan and colleagues identified two Escherichia coli noncoding RNAs, OxyS and DsrA, which could initiate feeding RNAi effect in C. elegans [39]. OxyS, a noncoding

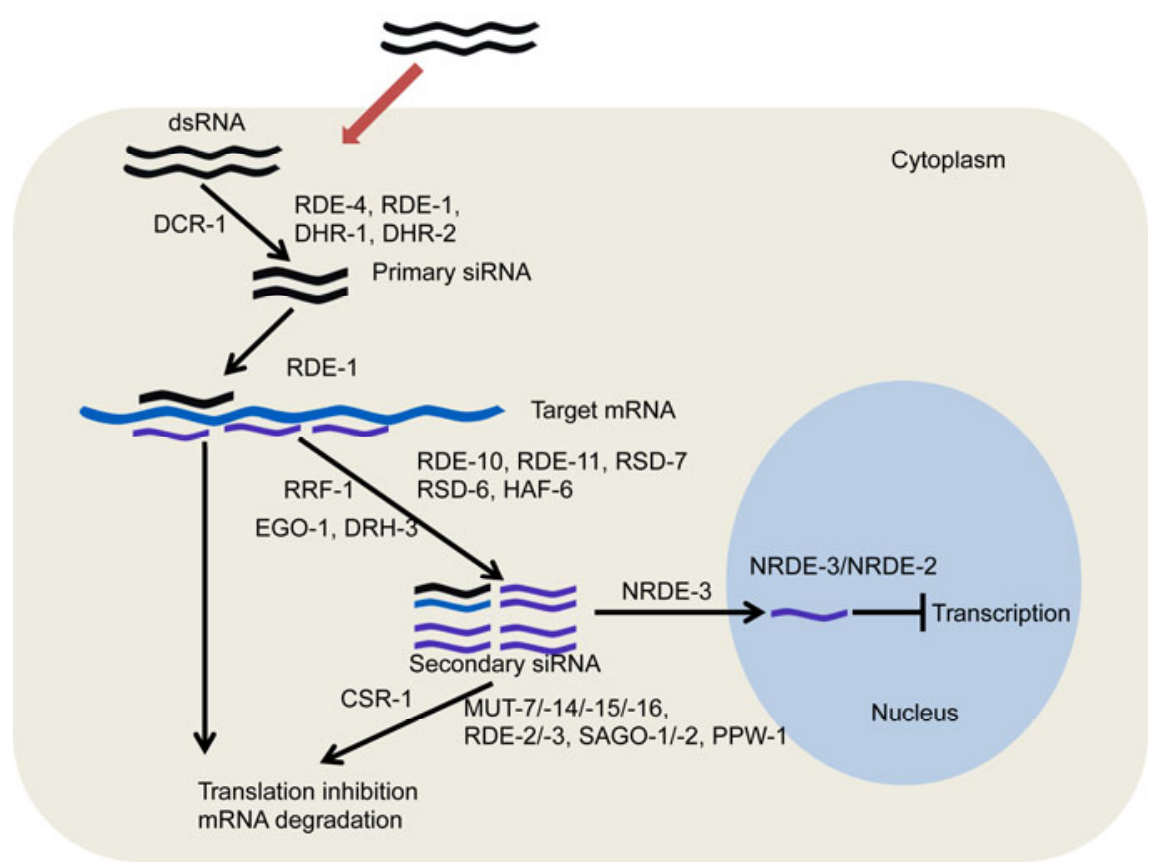

Figure 2 (Color online) Proposed molecular pathway for environmental RNA interference triggered by exogenous RNAs in C. elegans. 
RNA expressed in $E$. coli under oxidative stress, impaired $C$. elegans olfactory ability by downregulating a chemosensory gene che-2. DsrA, a noncoding RNA expressed in E. coli under $25^{\circ} \mathrm{C}$ or below, decreased $C$. elegans longevity by suppressing diacylglycerol lipase gene $F 42 G 9.6$, Other bacteria such as Bacillus mycoides might also utilize noncoding RNAs to interfere with gene expression in C. elegans [39]. These results demonstrated that $E$. coli noncoding RNAs could regulate gene expression and physiological conditions of $C$. elegans and indicated that noncoding RNAs might have interspecies ecological roles with natural feeding RNAi $[39,40]$.

OxyS and che-2 have 17 bp complementary sequences, and the inhibition of che-2 by OxyS requires alg-1, rde-4, and the ABC transporter gene haf-2. DsrA and F42G9.6 share a stretch of $27 \mathrm{nt}$ sequence. For DsrA, rde-4 and haf-6 were required for its effect on F42G9.6. It was surprising that multiple other genes previously identified in the environmental RNAi pathway including sid-1 and sid-2 might be irrelevant or redundant for the RNAi effect of OxyS and DsrA [39]. It is possible that noncoding RNAs in real nature actually utilize an overlapping yet distinct molecular pathway from artificial dsRNAs in environmental RNAi.

\section{Environmental RNAi in other animals}

Besides C. elegans, environmental RNAi has been observed in a wide range of animal species [41,42]. Environmental RNAi is effective in a number of animal- and plant-parasitic nematodes [42-44]. In the planarian nematode Dugesia japonica, soaking of animals in a dsRNA solution could trigger gene knockdown both in cells with direct contact to the environment and in tissues inside the body [45]. Feeding bacteria expressing dsRNA to another planarian nematode Schmidtea mediterranea could also trigger an RNAi effect [46]. Efficient environmental RNAi could be achieved in the filarial nematode Brugia malayi by application of exogenous siRNA or dsRNA both in vitro (cultured separately from the host) and in vivo (in an intermediate host, the mosquito Aedes aegypti) [47].

Environmental RNAi by feeding or soaking has also been demonstrated in species of insects [48]. In the model organism D. melanogaster, soaking embryos in dsRNA solutions could trigger environmental RNAi effects [49]. There was even one report about induction of robust RNAi in Drosophila S2 cells with E. coli expressing dsRNA, and the authors believed that phagocytic uptake was underlying this RNAi effect [50]. However, there has been no report of environmental RNAi in Drosophila larvae or adults. Oral dsRNA delivery initiated RNAi effect of gene silencing in apple moth (Epiphyas postvittana) larvae but not in the oriental leafworm moth (Spodoptera litura) [51], although a previous study showed that dsRNA injected could silence of midgut aminopeptidase $\mathrm{N}$ in Spodoptera litura [52].
Environmental RNAi has also been observed in hydra, which belongs to cnidarians, a sister group to the bilaterians (vertebrates, insects, nematodes). Repeated feedings of dsRNA-expressing bacteria specifically suppressed the expression of target gene in hydra, and eventually phenocopied the phenotype shown in mutants of the target gene [53].

Unlike $C$. elegans and other invertebrates, mammals have complex digestive system and circulatory system, which may make them infeasible for environmental RNAi. Nevertheless a successful example has been reported, in which recombinant nonpathogenic short-hairpin RNA expressing E. coli suppressed the expression of an oncogene, $C T N N B 1$, in the intestinal epithelium and in human colon cancer xenografts in mice [54]. Oral delivery of siRNA particles encapsulated with $\beta-1,3-D$-glucan, a component of yeast cell wall, could silence gene expression in mouse macrophages both in vitro and in vivo [55]. Both these examples are with engineered artificial delivery system of exogenous RNAs.

\section{Features and perspectives of animal environmental RNAi}

Mechanisms for the entry of environmental dsRNA into intestinal cells and the subsequent intercellular exporting and importing of environmental RNAi signal have only been investigated systematically in $C$. elegans for several years. Exact roles of sid- 1 and sid-2 in uptaking of environmental dsRNA by intestinal cells are still elusive $[7,8,12,14]$. Is SID-1 a channel and SID-2 a receptor for dsRNA, or both of them just bind to dsRNA and promote the subsequent endocytosis of dsRNA? Genes such as sid-3 and sid-5 related to endocytosis and exocytosis have been linked to environmental RNAi in C. elegans and Drosophila cells, although a direct role of endocytosis and exocytosis in the uptaking, importing, and exporting of exogenous RNAs has not been assigned [15,16]. Further investigations must be performed to answer these questions.

While the general frame of functional mechanism for RNA interference is relatively conserved in multicellular organisms, a lot of details associated with environmental RNAi are not well conserved. C. briggsae, a sister species of C. elegans, is responsible for injected dsRNA, while it is incompetent in environmental RNAi by feeding or soaking [8]. Sequence divergence of SID-2 protein between $C$. elegans and $C$. briggsae may be underneath the differential sensitivity to environmental RNAi between these two species. C. briggsae SID-2 has relatively low sequence similarity to $C$. elegans SID-2, and this is especially the case for their outmembrane $\mathrm{N}$ termini, which share only $23 \%$ identity between them. Expressing the C. elegans sid-2::gfp fusion in $C$. briggsae resulted its sensitivity to environmental RNAi triggered by dsRNA [8]. On the other hand though, $C$. briggsae is sensitive to natural feeding RNAi initiated by $E$. 
coli noncoding RNAs such as OxyS and DsrA, and actually sid-1 and sid-2 are irrelevant or redundant for this natural feeding RNAi phenomenon [39]. Both mice and human genomes harbor two sid-1 homologs, SidT1 and SidT2, and SidT1 has a role in the uptake of dsRNA by human cells, as blocking or knocking down of SidT1 resulted in defect in internalization of cholesterol-conjugated siRNA by human hepatocyte in vitro [56].

A comparative analysis of the draft genome of the filarial nematode Brugia malayi with the C. elegans genome revealed that there were apparently no sid-1 and sid-2 homologs in Brugia malayi despite that this parasitic nematode is competent for environmental RNAi [57]. A survey has been performed for orthologs of 77 Caenorhabditis elegans RNAi pathway proteins in 13 nematode species (transcriptomes of Ancylostoma caninum and Oesophagostomum dentatum, genomes of Trichinella spiralis, Ascaris suum, Brugia malayi, Haemonchus contortus, Meloidogyne hapla, Meloidogyne incognita, Pristionchus pacificus, as well as four Caenorhabditis species $C$. brenneri, $C$. briggsae, $C$. japonica and C. remanei) [58]. The result revealed that most of the $C$. elegans genes responsible for uptaking and spreading of the exogenous dsRNA are absent from parasitic species, including those nematodes competent for environmental RNAi [58]. It is possible that these genes might be fast evolving, or animals might have evolved alternative pathways for the uptaking of environmental dsRNA.

The functional mechanism of environmental RNAs in animals or even in C. elegans is not fully understood neither. For example, defined roles of $\mathrm{ABC}$ transporters in environmental RNAi are missing [33-35]. These transporters may import some small molecules crucial for the generation of secondary siRNA in C. elegans, or they may be responsible for generating optimal condition (e.g. pH) for the effect of RNAi, and these possibilities have to be tested.

The functional pathway for exogenous environmental RNAi shares many components with that of the endogenous siRNA in C. elegans, and actually they may compete for shared factors such as secondary Argonautes [38]. Is there any regulatory mechanism to coordinate the effects of exogenous and endogenous RNAi? Can C. elegans actually differentiate exogenous RNAs from endogenous siRNAs? These intriguing questions demand vigorous researches. Functional pathway for environmental RNAi also shows some divergence, as secondary Argonautes are poorly conserved among nematodes with the nuclear AGO NRDE-3 missing in any parasitic nematode [58]. Functional mechanism of environmental RNAi shows divergence even within the C. elegans species. C. elegans wild isolate CB4856 is resistant to germline RNAi with exogenous dsRNA, and this resistance is linked to a single significant locus on chromosome I that includes the Argonaute gene $p p w-1$ [59].

Functional mechanisms underlying environmental RNAi were basically elucidated with synthesized or recombinantly expressed dsRNAs. Natural feeding RNAi triggered by noncoding RNAs OxyS and DsrA utilize an overlapping but distinct molecular pathway from exogenous dsRNAs [39]. Are dsRNAs, or instead noncoding RNAs such as OxyS and DsrA, the major catalog of RNAs in real nature for triggering environmental RNAi? Careful and systematical investigations have to be carried out to answer this important question.

\section{Environmental RNAi in a broader sense}

Dependent on how we define environmental RNAi, any phenomenon related to the interfering of gene expression by exogenous RNA could be considered as environmental RNAi in a broader sense. It is highly possible that interspecies RNAs may take part in the interactions between parasites (or even infectious bacteria) and their hosts. Although RNAi effect has been observed in parasitic animals with artificially administrated exogenous RNAs, essentially there is still no report about "real" environmental RNAi elicited between parasites and hosts by their own RNAs. To stretch it further, even certain interactions between viruses specifically RNA viruses and their hosts may be considered as environmental RNAi, as genomic RNAs or RNA transcripts of virus often manipulate gene expression of host cells via RNAi pathway. More details about virus, host cell, and RNAi could be found in several review articles [60-62].

There is another more intriguing example of environmental RNAi if you like. Investigators recently reported that exogenous plant miRNAs were present in the sera and tissues of mice and human [63]. mir-168a, which is abundant in rice, was detected in microvesicles of animals after having taken rice as food. Plant mir-168a could silence lowdensity lipoprotein receptor adapter protein 1 (LDLRAP1) expression in mice [63]. Because plants and mammals are different in their functional mechanisms of microRNAs, and mammals have complex digestive system, it would be more convincing for researchers to reveal the uptaking/importing and the functional mechanisms of these exogenous plant microRNAs. It is already known that certain cells in mammals such as peripheral blood mononuclear cells can generate exosomes or microvesicles packed with RNAs inside [64]. Some miRNAs have been identified in these exosomes and they have been shown to have intercellular gene regulatory effects [65]. This could be viewed as a kind of systemic RNAi in mammals, but whether exogenous small RNAs could utilize these microvesicles for intercellular transportation remains to be examined.

\section{Research and biotechnology application of environmental RNAi}

Feeding dsRNA-expressing E. coli can be conveniently applied in C. elegans for knocking down gene expression of individual genes, or at whole-genome scale, to identify 
candidate genes involved in specific biological events [66]. Whole-genome RNAi feeding libraries have been developed, and many successful systematic RNAi screenings have been performed [67-71]. In C. elegans, feeding RNAi can also be fine tuned to knockdown gene expression for a specific developmental stage [72]. Systematic RNAi screening also enables the application of reverse genetics in Drosophila S2 cells, since these cells can take up dsRNA from their culture medium [73,74].

A prominent field for application of environmental RNAi is in the control of pests of animals and plants. Soaking in dsRNA solutions could result in environmental RNAi effect in Schistosoma mansoni, a parasitic worm of human [75]. Multiple successful examples have been reported about exogenous dsRNA delivered from transgenic plants resulting in gene silencing in nematodes such as Meloidogyne species (root-knot nematodes)[76]. Ingestion of dsRNAs conditioned artificial diet triggered RNAi in the western corn rootworm (WCR, Diabrotica virgifera virgifera LeConte). Transgenic expressing dsRNAs against WCR gene in corn plants also showed protection to the crop again WCR feeding damage [77]. When cotton bollworm (Helicoverpa armigera) larvae were fed with plant material expressing dsRNA specific to a cytochrome P450 gene of cotton bollworm, decreased expression of this gene and retarded larval growth were observed [78]. Transgenic plants expressing dsRNA targeting to specific animals may be a general strategy to control agriculture pests.

Since the discovery of RNAi pathway, lot efforts have been made to develop RNAi-based therapeutics [66,79-81]. Attempts to apply short-hairpin RNA expressed E. coli for the suppressing oncogene in mice, deliver orally siRNA particles to silence gene expression in mouse macrophages, and inject siRNAs in saline solution for the knocking down of disease related genes are essentially application of environmental RNAi for the treatment for human diseases $[54,55,79]$. Although great optimism is hold for RNAibased therapy, hurdles have to be overcome to bring it into reality.

\section{Conclusion}

A substantial amount of knowledge in the field of environmental RNAi has been accumulated so far, while we are still just at the beginning to uncover all the mysteries associated with this phenomenon. One thing we need to point out is that environmental RNAi has also been found in plants and protozoans [6,82-85]. Environmental RNAi has been extensively applied in research with C. elegans, but its application in biotechnology and therapeutics requires further development. Research in environmental RNAi will no doubt be an active branch to continuously bring novel insights in the years to come, as the whole field of noncoding RNA research is moving fast forward [86-90].
The authors thank the members of the Ge Shan lab for discussion. This work was supported by the National Basic Research Program of China (2011CBA01103), National Natural Science Foundation of China (81171074, 91232702, and 31071132), and the Knowledge Innovation Project of CAS (1731112304041) to G. Shan.

1 Fire A, Xu S, Montgomery M K, et al. Potent and specific genetic interference by double-stranded RNA in Caenorhabditis elegans. Nature, 1998, 391: 806-811

2 Tabara H, Grishok A, Mello C C. RNAi in C. elegans: Soaking in the genome sequence. Science, 1998, 282: 430-431

3 Timmons L, Fire A. Specific interference by ingested dsRNA. Nature, 1998, 395: 854

4 Whangbo J S, Hunter C P. Environmental RNA interference. Trends Genet, 2008, 24: 297-305

5 Ruiz-Ferrer V, Voinnet O. Roles of plant small RNAs in biotic stress responses. Annu Rev Plant Biol, 2009, 60: 485-510

6 Kolev N G, Tschudi C, Ullu E. RNA interference in protozoan parasites: Achievements and challenges. Eukaryot Cell, 2011, 10: 1156-1163

7 Winston W M, Molodowitch C, Hunter C P. Systemic RNAi in C. elegans requires the putative transmembrane protein sid-1. Science, 2002, 295: 2456-2459

8 Winston W M, Sutherlin M, Wright A J, et al. Caenorhabditis elegans sid-2 is required for environmental RNA interference. Proc Natl Acad Sci USA, 2007, 104: 10565-10570

9 Timmons L, Tabara H, Mello C C, et al. Inducible systemic RNA silencing in Caenorhabditis elegans. Mol Biol Cell, 2003, 14: 29722983

10 Tijsterman M, May R C, Simmer F, et al. Genes required for systemic RNA interference in Caenorhabditis elegans. Curr Biol, 2004, 14: 111-116

11 Saleh M C, van Rij R P, Hekele A, et al. The endocytic pathway mediates cell entry of dsRNA to induce RNAi silencing. Nat Cell Biol, 2006, 8: 793-802

12 Feinberg E H, Hunter C P. Transport of dsRNA into cells by the transmembrane protein sid-1. Science, 2003, 301: 1545-1547

13 Jose A M, Smith J J, Hunter C P. Export of RNA silencing from $C$. elegans tissues does not require the RNA channel sid-1. Proc Natl Acad Sci USA, 2009, 106: 2283-2288

14 McEwan D L, Weisman A S, Hunter C P. Uptake of extracellular double-stranded RNA by SID-2. Mol Cell, 2012, 47: 746-754

15 Jose A M, Kim Y A, Leal-Ekman S, et al. Conserved tyrosine kinase promotes the import of silencing RNA into Caenorhabditis elegans cells. Proc Natl Acad Sci USA, 2012, 109: 14520-14525

16 Hinas A, Wright A J, Hunter C P. sid-5 is an endosome-associated protein required for efficient systemic RNAi in C. elegans. Curr Biol, 2012, 22: 1938-1943

17 Teo M, Tan L, Lim L, et al. The tyrosine kinase ACK1 associates with clathrin-coated vesicles through a binding motif shared by arrestin and other adaptors. J Biol Chem, 2001, 276: 18392-18398

18 Tabara H, Yigit E, Siomi H, et al. The dsRNA binding protein rde-4 interacts with rde-1, dcr-1, and a dexh-box helicase to direct RNAi in C. elegans. Cell, 2002, 109: 861-871

19 Knight S W, Bass B L. A role for the RNase III enzyme DCR-1 in RNA interference and germ line development in Caenorhabditis elegans. Science, 2001, 293: 2269-2271

20 Ketting R F, Fischer S E, Bernstein E, et al. Dicer functions in RNA interference and in synthesis of small RNA involved in developmental timing in C. elegans. Genes Dev, 2001, 15: 2654-2659

21 Tabara H, Sarkissian M, Kelly W G, et al. The rde-1 gene, RNA interference, and transposon silencing in C. elegans. Cell, 1999, 99: 123-132

22 Lu R, Yigit E, Li W X, et al. An RIG-I-Like RNA helicase mediates antiviral RNAi downstream of viral siRNA biogenesis in Caenorhabditis elegans. PLoS Pathog, 2009, 5: e1000286

23 Smardon A, Spoerke J M, Stacey S C, et al. ego-1 is related to RNA-directed RNA polymerase and functions in germ-line develop- 
ment and RNA interference in C. elegans. Curr Biol, 2000, 10: 169178

24 Sijen T, Fleenor J, Simmer F, et al. On the role of RNA amplification in dsRNA-triggered gene silencing. Cell, 2001, 107: 465-476

25 Sijen T, Steiner F A, Thijssen K L, et al. Secondary siRNAs result from unprimed RNA synthesis and form a distinct class. Science, 2007, 315: 244-247

26 Aoki K, Moriguchi H, Yoshioka T, et al. In vitro analyses of the production and activity of secondary small interfering RNAs in $C$. elegans. EMBO J, 2007, 26: 5007-5019

27 Matranga C, Pyle A M. Double-stranded RNA-dependent ATPase DRH-3: Insight into its role in RNA silencing in Caenorhabditis elegans. J Biol Chem, 2010, 285: 25363-25371

28 Claycomb J M, Batista P J, Pang K M, et al. The argonaute CSR-1 and its 22G-RNA cofactors are required for holocentric chromosome segregation. Cell, 2009, 139: 123-134

29 Yigit E, Batista P J, Bei Y, et al. Analysis of the C. elegans argonaute family reveals that distinct argonautes act sequentially during RNAi. Cell, 2006, 127: 747-757

30 Zhang C, Montgomery T A, Gabel H W, et al. mut-16 and other mutator class genes modulate $22 \mathrm{G}$ and $26 \mathrm{G}$ siRNA pathways in Caenorhabditis elegans. Proc Natl Acad Sci USA, 2011, 108: 1201-1208

31 Tops B B, Tabara H, Sijen T, et al. rde-2 interacts with mut-7 to mediate RNA interference in Caenorhabditis elegans. Nucleic Acids Res, 2005, 33: 347-355

32 Tijsterman M, Okihara K L, Thijssen K, et al. PPW-1, a PAZ/PIWI protein required for efficient germline $\mathrm{RNAi}$, is defective in a natural isolate of C. elegans. Curr Biol, 2002, 12: 1535-1540

33 Sundaram P, Echalier B, Han W, et al. ATP-binding cassette transporters are required for efficient RNA interference in Caenorhabditis elegans. Mol Biol Cell, 2006, 17: 3678-3688

34 Sundaram P, Han W, Cohen N, et al. Caenorhabditis elegans ABCRNAi transporters interact genetically with rde-2 and mut-7. Genetics, 2008, 178: 801-814

35 Zhang C, Montgomery T A, Fischer S E, et al. The Caenorhabditis elegans rde-10/rde-11 complex regulates RNAi by promoting secondary siRNA amplification. Curr Biol, 2012, 22: 881-890

36 Guang S, Bochner A F, Pavelec D M, et al. An Argonaute transports siRNAs from the cytoplasm to the nucleus. Science, 2008, 321: 537-541

37 Guang S, Bochner A F, Burkhart K B, et al. Small regulatory RNAs inhibit RNA polymerase II during the elongation phase of transcription. Nature, 2010, 465: 1097-1101

38 Yigit E, Batista P J, Bei Y, et al. Analysis of the C. elegans Argonaute family reveals that distinct Argonautes act sequentially during RNAi. Cell, 2006, 127: 747-757

39 Liu H, Wang X, Wang H D, et al. Escherichia coli noncoding RNAs can affect gene expression and physiology of Caenorhabditis elegans. Nat Commun, 2012, 3: 1073

40 Wootton L. Microbial ecology: Non-coding RNAs make E. coli unpalatable. Nat Rev Microbiol, 2012, 10: 732

41 Jose A M, Hunter C P. Transport of sequence-specific RNA interference information between cells. Annu Rev Genet, 2007, 41: 305-330

42 Bellafiore S, Briggs S P. Nematode effectors and plant responses to infection. Curr Opin Plant Biol, 2010, 13: 442-448

43 Maule A G, McVeigh P, Dalzell J J, et al. An eye on RNAi in nematode parasites. Trends Parasitol, 2011, 27: 505-513

44 Lilley C J, Davies L J, Urwin P E. RNA interference in plant parasitic nematodes: A summary of the current status. Parasitology, 2012, 139: $630-640$

45 Orii H, Mochii M, Watanabe K. A simple "soaking method" for RNA interference in the planarian Dugesia japonica. Dev Genes Evol, 2003, 213: 138-141

46 Newmark P A, Reddien P W, Cebria F, et al. Ingestion of bacterially expressed double-stranded RNA inhibits gene expression in planarians. Proc Natl Acad Sci USA, 2003, 100: 11861-11865

47 Song C, Gallup J M, Day T A, et al. Development of an in vivo RNAi protocol to investigate gene function in the filarial nematode, Brugia malayi. PLoS Pathog, 2010, 6: e1001239
48 Bellés X. Beyond Drosophila: RNAi in vivo and functional genomics in insects. Annu Rev Entomol, 2010, 55: 111-128

49 Eaton B A, Fetter R D, Davis G W. Dynactin is necessary for synapse stabilization. Neuron, 2002, 34: 729-741

50 Rocha J J, Korolchuk V I, Robinson I M, et al. A phagocytic route for uptake of double-stranded RNA in RNAi. PLoS One, 2011, 6: e19087

51 Turner C T, Davy M W, MacDiarmid R M, et al. RNA interference in the light brown apple moth, Epiphyas postvittana (walker) induced by double-stranded RNA feeding. Insect Mol Biol, 2006, 15: 383391

52 Rajagopal R, Sivakumar S, Agrawal N, et al. Silencing of midgut aminopeptidase $\mathrm{N}$ of Spodoptera litura by double-stranded RNA establishes its role as Bacillus thuringiensis toxin receptor. J Biol Chem, 2002, 277: 46849-46851

53 Chera S, de Rosa R, Miljkovic-Licina M, et al. Silencing of the hydra serine protease inhibitor kazall gene mimics the human spink1 pancreatic phenotype. J Cell Sci, 2006, 119: 846-857

54 Xiang S, Fruehauf J, Li C J. Short hairpin RNA-expressing bacteria elicit RNA interference in mammals. Nat Biotechnol, 2006, 24: 697-702

55 Aouadi M, Tesz G J, Nicoloro S M, et al. Orally delivered siRNA targeting macrophage map4k4 suppresses systemic inflammation. Nature, 2009, 458: 1180-1184

56 Wolfrum C, Shi S, Jayaprakash K N, et al. Mechanisms and optimization of in vivo delivery of lipophilic siRNAs. Nat Biotechnol, 2007, 25: 1149-1157

57 Ghedin E, Wang S, Spiro D, et al. Draft genome of the filarial nematode parasite Brugia malayi. Science, 2007, 317: 1756-1760

58 Dalzell J J, McVeigh P, Warnock N D, et al. RNAi effector diversity in nematodes. PLoS Negl Trop Dis, 2011, 5: e1176

59 Pollard D A, Rockman M V. Resistance to germline RNAi in a Caenorhabditis elegans wild isolate exhibits complexity and non-additivity. G3 (Bethesda), 2013, doi: 10.1534/g3.113.005785

60 Li F, Ding S W. Virus counterdefense: Diverse strategies for evading the RNA-silencing immunity. Annu Rev Microbiol, 2006, 60: 503-531

61 Goic B, Saleh M C. Living with the enemy: Viral persistent infections from a friendly viewpoint. Curr Opin Microbiol, 2012, 15: 531537

62 Blake S J, Bokhari F F, McMillan N A. RNA interference for viral infections. Curr Drug Targets, 2012, 13: 1411-1420

63 Zhang L, Hou D, Chen X, et al. Exogenous plant mir168a specifically targets mammalian LDLRAP1: Evidence of cross-kingdom regulation by microRNA. Cell Res, 2012, 22: 107-126

64 Valadi H, Ekstrom K, Bossios A, et al. Exosome-mediated transfer of mRNAs and microRNAs is a novel mechanism of genetic exchange between cells. Nat Cell Biol, 2007, 9: 654-659

65 Gibbings D J, Ciaudo C, Erhardt M, et al. Multivesicular bodies associate with components of miRNA effector complexes and modulate miRNA activity. Nat Cell Biol, 2009, 11: 1143-1149

66 Shan G. RNA interference as a gene knockdown technique. Int J Biochem Cell Biol, 2010, 42: 1243-1251

67 Ashrafi K, Chang F Y, Watts J L, et al. Genome-wide RNAi analysis of Caenorhabditis elegans fat regulatory genes. Nature, 2003, 421: 268-272

68 Kim J K, Gabel H W, Kamath R S, et al. Functional genomic analysis of RNA interference in C. elegans. Science, 2005, 308: 1164-1167

69 Samuelson A V, Klimczak R R, Thompson D B, et al. Identification of Caenorhabditis elegans genes regulating longevity using enhanced RNAi-sensitive strains. Cold Spring Harb Symp Quant Biol, 2007, 72: 489-497

70 Parry D H, Xu J, Ruvkun G. A whole-genome RNAi screen for $C$. elegans miRNA pathway genes. Curr Biol, 2007, 17: 2013-2022

71 Mabon M E, Mao X, Jiao Y, et al. Systematic identification of gene activities promoting hypoxic death. Genetics, 2009, 181: 483-496

72 Shan G, Walthall W W. Copulation in C. elegans males requires a nuclear hormone receptor. Dev Biol, 2008, 322: 11-20

73 Caplen N J, Fleenor J, Fire A, et al. dsRNA-mediated gene silencing in cultured Drosophila cells: A tissue culture model for the analysis of RNA interference. Gene, 2000, 252: 95-105 
74 D'Ambrosio M V, Vale R D. A whole genome RNAi screen of Drosophila $\mathrm{S} 2$ cell spreading performed using automated computational image analysis. J Cell Biol, 2010, 191: 471-478

75 Boyle J P, Wu X J, Shoemaker C B, et al. Using RNA interference to manipulate endogenous gene expression in Schistosoma mansoni sporocysts. Mol Biochem Parasitol, 2003, 128: 205-215

76 Huang G, Allen R, Davis E L, et al. Engineering broad root-knot resistance in transgenic plants by RNAi silencing of a conserved and essential root-knot nematode parasitism gene. Proc Natl Acad Sci USA, 2006, 103: 14302-14306

77 Baum J A, Bogaert T, Clinton W, et al. Control of coleopteran insect pests through RNA interference. Nat Biotechnol, 2007, 25: 1322-1326

78 Mao Y B, Cai W J, Wang J W, et al. Silencing a cotton bollworm p450 monooxygenase gene by plant-mediated RNAi impairs larval tolerance of gossypol. Nat Biotechnol, 2007, 25: 1307-1313

79 Shan G, Jin P. RNA interference to treat human diseases. In: Begley T, ed. Wiley Encyclopedia of Chemical Biology. Vol. 4. Hoboken: John Wiley \& Sons, 2009. 241-251

80 Shan G, Li Y, Zhang J, et al. A small molecule enhances RNA interference and promotes microRNA processing. Nat Biotechnol, 2008, 26: 933-940

81 Gavrilov K, Saltzman W M. Therapeutic siRNA: Principles, chal- lenges, and strategies. Yale J Biol Med, 2012, 85: 187-200

82 Fu D, Uauy C, Blechl A, et al. RNA interference for wheat functional gene analysis. Transgenic Res, 2007, 16: 689-701

83 Baulcombe D. RNA silencing in plants. Nature, 2004, 431: 356-363

84 Travella S, Klimm T E, Keller B. RNA interference-based gene silencing as an efficient tool for functional genomics in hexaploid bread wheat. Plant Physiol, 2006, 142: 6-20

85 Ullu E, Tschudi C, Chakraborty T. RNA interference in protozoan parasites. Cell Microbiol, 2004, 6: 509-519

86 Lin M, Wu J, Shan G. Noncoding RNAs: Different roles in tumorigenesis. Chin Sci Bull, 2012, 57: 959-965

$87 \mathrm{Hu} \mathrm{S}, \mathrm{Wu}$ J, Chen L, et al. Signals from noncoding RNAs: Unconventional roles for conventional pol III transcripts. Int $\mathrm{J}$ Biochem Cell Biol, 2012, 44: 1847-1851

88 Yang L, Meng Y, Bao C, et al. Robustness and backbone motif of a cancer network regulated by miR-17-92 cluster during the G1/S transition. PLoS One, 2013, 8: e57009

89 Lee J T, Bartolomei M S. X-inactivation, imprinting, and long noncoding RNAs in health and disease. Cell, 2013, 152: 1308-1323

90 Castel S E, Martienssen R A. RNA interference in the nucleus: Roles for small RNAs in transcription, epigenetics and beyond. Nat Rev Genet, 2013, 14: 100-112

Open Access This article is distributed under the terms of the Creative Commons Attribution License which permits any use, distribution, and reproduction in any medium, provided the original author(s) and source are credited. 